\title{
On scale-free and poly-scale behaviors of random hierarchical networks
}

\author{
V.A. Avetisov ${ }^{1}$, A.V. Chertovich ${ }^{2}$, S.K. Nechaev ${ }^{3 *}$, O.A. Vasilyev ${ }^{4,5}$ \\ ${ }^{1}$ N.N. Semenov Institute of Chemical Physics of the Russian Academy of Sciences, 1199911, Moscow, Russia \\ ${ }^{2}$ Physics Department, Moscow State University, 119992 Moscow, Russia \\ ${ }^{3}$ LPTMS, Université Paris Sud, 91405 Orsay Cedex, France \\ ${ }^{4}$ Max-Planck-Institut für Metallforschung, Heisenbergstr. 3, D-70569 Stuttgart, Germany \\ ${ }^{5}$ Institut für Theoretische und Angewandte Physik, \\ Universität Stuttgart, Pfaffenwaldring 57, D-70569 Stuttgart, Germany
}

(Dated: November 6, 2018)

\begin{abstract}
In this paper the question about statistical properties of block-hierarchical random matrices is raised for the first time in connection with structural characteristics of random hierarchical networks obtained by mipmapping procedure. In particular, we compute numerically the spectral density of large random adjacency matrices defined by a hierarchy of the Bernoulli distributions $\left\{q_{1}, q_{2}, \ldots\right\}$ on matrix elements, where $q_{\gamma}$ depends on hierarchy level $\gamma$ as $q_{\gamma}=p^{-\mu \gamma}(\mu>0)$. For the spectral density we clearly see the free-scale behavior. We show also that for the Gaussian distributions on matrix elements with zero mean and variances $\sigma_{\gamma}=p^{-\nu \gamma}$, the tail of the spectral density, $\rho_{G}(\lambda)$, behaves as $\rho_{G}(\lambda) \sim|\lambda|^{-(2-\nu) /(1-\nu)}$ for $|\lambda| \rightarrow \infty$ and $0<\nu<1$, while for $\nu \geq 1$ the power-law behavior is terminated. We also find that the vertex degree distribution of such hierarchical networks has a poly-scale fractal behavior extended to a very broad range of scales.
\end{abstract}

PACS numbers: 05.40.a, 87.15.hg

\section{INTRODUCTION}

The determination of the structural organization of the complex system with statistical disorder refers often to a "network paradigm", implying the investigation of topological and statistical characteristics of the contact map between the system elements. Analytical approaches to this task commonly use the well-defined relationship between the spectral properties of the adjacency (connectivity) matrix, which codes the contacts, and a particular topological structure of the network. In the case of uncorrelated random networks (graphs) known as Erdös-Rényi (ER) graphs 1], there exists a bunch of methods for analytical study of spectra of the adjacency matrix - from mathematically rigorous to less rigorous. The last ones are often borrowed from the statistical mechanics of disordered systems. Among the most used are the replica [2] or the Flory mean-field [3] approaches.

In the last decade, an explosive growth of computational power has allowed to accumulate a large body of data on statistical and topological characteristics of real networks of diverse nature. The "network paradigm" is currently spread from protein folding, intermolecular contacts in biopolymers, genetic maps and cell metabolism, up to natural networks (including world wide web, various ecological, social, financial and economic entities, etc.), statistical data analysis, and even to Bose-Einstein condensation. Many of these topics are reviewed in [4]. In majority of cases it turned out that the statistical characteristics of real network topology, as well as the spectral properties of the adjacency matrices, essentially differ from the characteristics of random ER-graphs. For example, it has been found that the probability distributions of typical topological characteristics (e.g. the vertex degree distribution, or the clustering coefficient) have the power-law tails for many real networks, in contrast to the exponential tails for ERgraphs. Thus, the networks of such topology were stood out for a class of the "scale-free" networks just because of the power-law behavior of some observables. In practice, the network is often referred to belong to the "scalefree" class if the vertex degree distribution (i.e. the distribution of nearest-neighboring contacts over the network vertices) has the power-law tail. However in general setting dealing with the analysis of the spectral density of the adjacency matrix we shall understand under the "scale-free"-behavior the existence of the power-law tail in the spectral density. The topological characteristics of these networks, such as, for example, the vertex degree, have extremely wide distributions exceeding the corresponding distributions for ER-graphs by orders of magnitude. Such anomalously wide distributions we shall call "poly-scaled" to distinct them out of the "scale-free" behavior.

The main idea of our work consists in the construction of networks with block-hierarchical adjacency matrices resulting from an appropriate randomization of the standard Parisi matrix - one of the key objects in the theory of

\footnotetext{
* Also at: P.N. Lebedev Physical Institute of the Russian Academy of Sciences, 119991, Moscow, Russia
} 
spin glasses (see [5] for example). In other words, we study below the random block-hierarchical (RBH) networks. By the behavior of the spectral density of adjacency matrices, the RBH networks fall into the "scale-free" class, but in majority of cases, they have "poly-scaled" distribution of the vertex degree.

It would not be an exaggeration to say that the block-hierarchical "ordering" is rather typical than exceptional for many complex systems which are both random and multi-scaled. The examples of such networks can be easily find in different areas of mathematics, physics and biology: from chaotic maps in Hamiltonian systems [6, 7] to condensed (globular) structures of polyelectrolyte chains [8] and hierarchical organization of biopolymers [9]. We anticipate our consideration of spectral properties of the $\mathrm{RBH}$ adjacency matrices with a generic example of possible realization of a block-hierarchical contact map in a globular phase of unknotted ring polymer molecules with topological interactions.

It is known that the non-phantomness of a polymer chain causes two types of interactions: i) volume interactions vanishing for infinitely thin chains, and ii) topological interactions, which present even for chains of zero thickness. For sufficiently high temperatures, a polymer molecule strongly fluctuates without reliable thermodynamic state called a coil state. However for temperatures below some critical value, $\theta$, the polymeric chain exhibits a dense weakly fluctuating globular (drop-like) structure [10, 11]. In classical works 10, 11] devoted to the coil-to-globule phase transition without topological constraints, it has been shown that, for $T<\theta$, the globular state can be described by using just only two- and three-body interaction constants: $B=b \frac{T-\theta}{\theta}<0$ and $C=$ const $>0$ (see [12, 13]). The approach developed in [10, 11] is regarded as the basic one in modern statistical theory of collapsed polymeric state.

The topological constraints in the globular phase of an unknotted macromolecule act the part of repulsive interactions. For the temperatures below $\theta$-point (i.e. in a poor solvent), against the temperature and the energy of volume interactions there exists certain scale of the chain length, $g^{*}$, such that the chains longer than $g^{*}$ collapse. Taking an enough long chain, we can define these $g^{*}$-unit parts as new "block monomers" (or "folds of minimal scale"). In the Fig 1 they are denoted as the 1st level folds. Sufficiently long parts of the chain with several folds of minimal scale should again "collapse in itself", i.e. they should form the 2 nd level folds, if other chain parts do not interfere with it. The chain of such new sub-blocks of the 2nd level folds collapses again forming the 3rd level folds, and so on... This block-hierarchical folding is completed when the initial chain units are united into one fold of the largest scale. Three first consecutive steps of such a process are shown in Fig 1a. Note that the line representing the chain folded by this way resembles the 3D-analogue of the well known self-similar Peano curve. The specific feature of the crumpled globule consists in the fact that different chain parts are not entangled with each others, completely fill the allowed volume of space and are "collapsed in themselves" starting from the characteristic scale $g^{*}$.

The scale of the fold, shown by hues of gray in Fig 1h, can be considered as a cutoff for the interaction distance between $g^{*}$-block monomers in the current fold. The values $t_{\gamma}^{(n)}$ can be thought as interaction constants between these $g^{*}$-block monomers in the $\gamma$-level fold and, thus, they constitute the contact map $T$ depicted in Fig 1 b.

In [14] and later, more rigorously, in [15] it has been argued that the absence of knots in a densely packed polymer ring causes a very peculiar fractal structure of the chain trajectory, strongly affecting all thermodynamic properties of the macromolecule in the globular phase. The corresponding structure of a collapsed unknotted polymer ring was called a crumpled globule. The chain trajectory in the crumpled globule densely fills the volume such that all part of the chain become segregated from each other in a broad region of scales. This model has been used later (see, for instance, [16, 17]) to describe self-similar hierarchical organization in some biopolymers, like DNA and chromatin.

Below we discuss some topological properties of block-hierarchical random networks. On the basis of obtained results we propose the new way of building of hierarchical networks with scale-free and poly-scaled properties in a memoryless locally uniform way. This method does not demand the control of the current state of the network.

\section{II. "HEAVY TAILS" IN THE SPECTRAL DENSITY OF RANDOM BLOCK-HIERARCHICAL ADJACENCY MATRICES}

Let us start with a description of generic procedure of the $\mathrm{RBH}-$ network construction. Taking $N$ points as potential vertices of our forthcoming network, we raise a hierarchical network by connecting the vertices by edges in a specific way. In the outset, we introduce an ensemble of the $N \times N$ adjacency matrices, $T$; each of them encoding the edges between connected vertices in a network realization. Namely, the element $T_{i, j}$ of $T$ is 1 if the vertices $i$ and $j$ are directly connected, otherwise $T_{i, j}=0$. We consider the adjacency matrix in very peculiar form of a $p$-adic translation-noninvariant Parisi matrix. This matrix is shown in Fig 10 for $p=2$. Obviously, $T_{i, j}=T_{j, i}$ and $T_{i, i}=0$. 


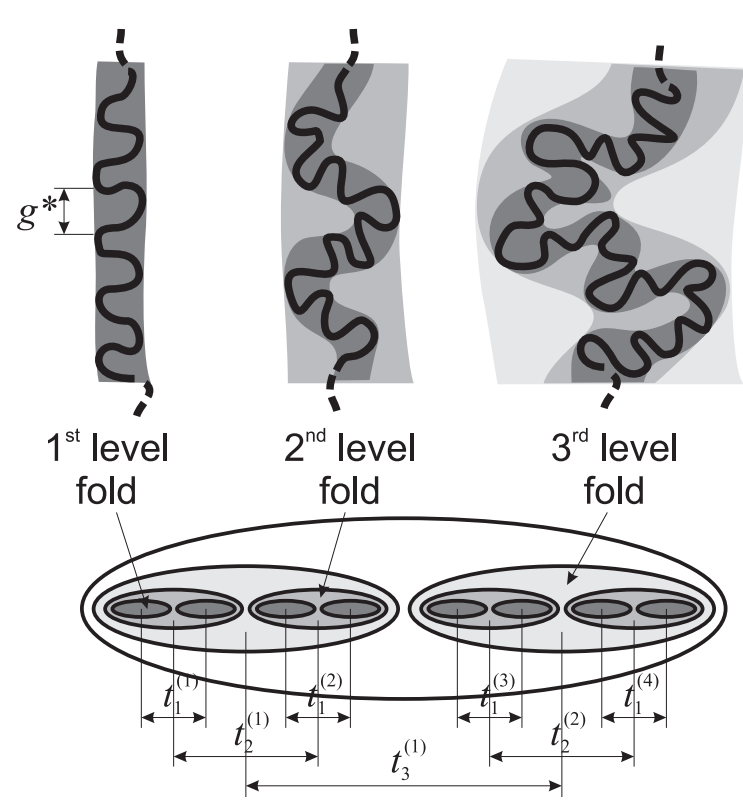

(a)

\begin{tabular}{|c|c|c|c|c|c|c|c|}
\hline 0 & $t_{1}^{(1)}$ & $t_{2}^{(1)}$ & $t_{2}^{(1)}$ & $t_{3}^{(1)}$ & $t_{3}^{(1)}$ & $t_{3}^{(1)}$ & $t_{3}^{(1)}$ \\
\hline$t_{1}^{(1)}$ & 0 & $t_{2}^{(1)}$ & $t_{2}^{(1)}$ & $t_{3}^{(1)}$ & $t_{3}^{(1)}$ & $t_{3}^{(1)}$ & $t_{3}^{(1)}$ \\
\hline$t_{2}^{(1)}$ & $t_{2}^{(1)}$ & 0 & $t_{1}^{(2)}$ & $t_{3}^{(1)}$ & $t_{3}^{(1)}$ & $t_{3}^{(1)}$ & $t_{3}^{(1)}$ \\
\hline$t_{2}^{(1)}$ & $t_{2}^{(1)}$ & $t_{1}^{(2)}$ & 0 & $t_{3}^{(1)}$ & $t_{3}^{(1)}$ & $t_{3}^{(1)}$ & $t_{3}^{(1)}$ \\
\hline & $t_{3}^{(1)}$ & $t_{3}^{(1)}$ & $t_{3}^{(1)}$ & 0 & $t_{1}^{(3)}$ & $t_{2}^{(2)}$ & $t_{2}^{(2)}$ \\
\hline & $t_{3}^{(1)}$ & $t_{3}^{(1)}$ & $t_{3}^{(1)}$ & $t_{1}^{(3)}$ & 0 & $t_{2}^{(2)}$ & $t_{2}^{(2)}$ \\
\hline & $t_{3}^{(1)}$ & $t_{3}^{(1)}$ & $t_{3}^{(1)}$ & $t_{2}^{(2)}$ & $t_{2}^{(2)}$ & 0 & $t_{1}^{(4)}$ \\
\hline$t_{3}^{(1)}$ & $t_{3}^{(1)}$ & $t_{3}^{(1)}$ & $t_{3}^{(1)}$ & $t_{2}^{(2)}$ & $t_{2}^{(2)}$ & $t_{1}^{(4)}$ & 0 \\
\hline
\end{tabular}

(b)

Figure 1: (a) Three subsequent stages of the construction of hierarchical contact map for the "crumpled globule" (see the text for details); (b) Block-hierarchical $p$-adic Parisi matrix $T(p=2)$.

All matrix elements, $t_{\gamma}^{(n)}$, are the Bernoulli distributed random variables:

$$
t_{\gamma}^{(n)}= \begin{cases}1 & \text { with the probability } q_{\gamma} \\ 0 & \text { with the probability } 1-q_{\gamma}\end{cases}
$$

where $\gamma$ counts the hierarchy levels $\left(1 \leq \gamma \leq \gamma_{\max } \equiv \Gamma\right)$ and $n$ enumerates different blocks corresponding to a given hierarchy level $\gamma$ (see Fig!1 ). Note that the probability $q_{\gamma}$ does not depend on $n$. The full ensemble of $N \times N$ matrices $T$, where $N=p^{\Gamma}$, is completely determined by the set of probabilities, $\{Q\}=\left\{q_{1}, q_{2}, \ldots, q_{\Gamma}\right\}$. Thus, the elements $T_{i, j}$, being the random variables, are hierarchically organized in probabilities. Below we consider the set of probabilities, $\{Q\}$, with $q_{\gamma}=p^{-\mu \gamma}(\mu>0)$.

The systematic study of statistical properties of ensembles of random graphs (networks) deals with the investigation of the spectral properties of a graph adjacency matrix [18, 19]. Let $\lambda_{i}(1 \leq i \leq N)$ be the eigenvalue of the adjacency matrix. The spectral density of the ensemble of random symmetric adjacency matrices is defined in the standard way,

$$
\rho(\lambda)=\frac{1}{N} \sum_{i=1}^{N}\left\langle\delta\left(\lambda-\lambda_{i}\right)\right\rangle_{\left\{q_{1}, q_{2}, \ldots, q_{\Gamma}\right\}}
$$

where $\langle\ldots\rangle_{\left\{q_{1}, q_{2}, \ldots q_{n}\right\}}$ denotes the averaging over the distributions of the matrix elements, $t_{\gamma}^{(n)}$.

Computing numerically the spectral density, $\rho(\lambda)$, of networks with block-hierarchical adjacency matrices, we found that the tails of the spectral density $\rho(\lambda)$ follow a power-law asymptotic behavior $\rho(\lambda) \sim|\lambda|^{-\chi}$ with the exponent $\chi=\chi(\mu)$. The sample plots of the spectral density $\rho(\lambda)$ are shown for $N=256,2048$ and $\mu=0.2$ in Fig 2 in semi-log coordinates. The corresponding $\log -\log$ plot of the left- and right-hand tails of the spectral density for $N=256$ is drawn in Fig 2 $\mathrm{b}$. It is interesting to note that the right-hand tail of $\rho(\lambda)$, while demonstrating the same behavior, is worse averaging.

Some analytic arguments supporting the found power-law behavior of the spectral statistics of the RBH- graphs can be brought by means of the spectral density, $\rho_{G}(\lambda)$, of the Gaussian ensemble of the Parisi matrices $T$, where the Bernoulli distributions on matrix elements of $T$ are replaced by the Gaussian distributions with zero means and a set of variances $\{\mathcal{S}\}=\left\{\sigma_{1}, \sigma_{2}, \ldots, \sigma_{\Gamma}\right\}, \sigma_{\gamma}=p^{-\nu \gamma}(\nu>0)$. To get the spectral density $\rho_{G}(\lambda)$, note that the eigenvalues of the standard (i.e. translation-invariant) Parisi matrix with $T_{i, i}=0$ can be expressed in terms of matrix elements 


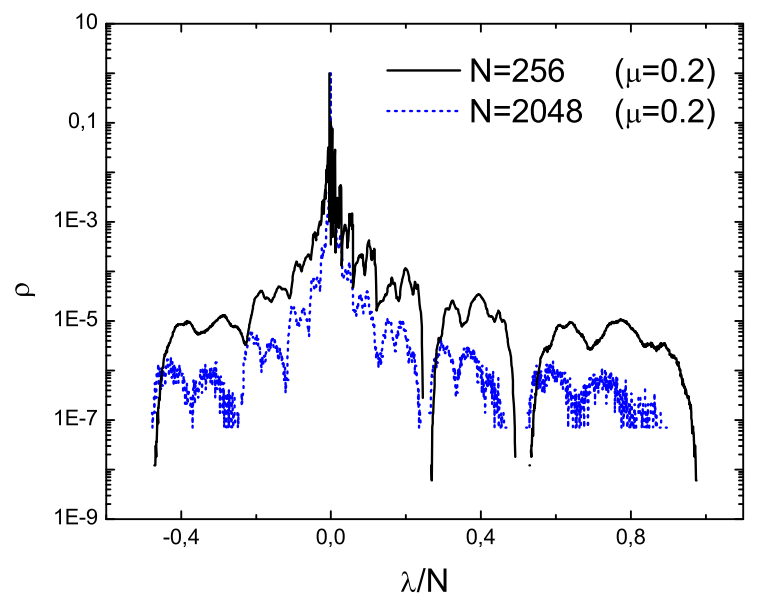

(a)

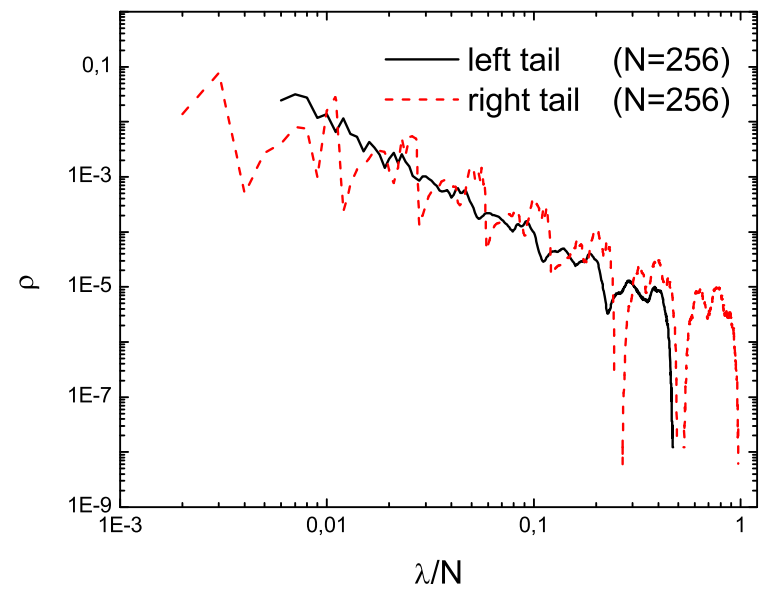

(b)

Figure 2: Spectral density $\rho(\lambda)$ for $\mu=0.2$ : (a) semi-log plot of the full distribution for $N=256$ (solid line) and $N=2048$ (dashed line), (b) the left- and right-hand tails of $\rho(\lambda)$ for $N=256$ in log-log coordinates.

$t_{\gamma}^{(n)} \equiv t_{\gamma}$ as follows $([20]):$

$$
\lambda_{\gamma}=p^{\gamma} t_{\gamma}-\left(1-p^{-1}\right) \sum_{\gamma^{\prime}=1}^{\gamma} p^{\gamma^{\prime}} t_{\gamma^{\prime}} \quad(\gamma=1, \ldots, \Gamma)
$$

The eigenvalue $\lambda_{\gamma}$ is $p^{\Gamma-\gamma}$ times degenerated $(\gamma=1, \ldots, \Gamma)$. In addition there is one extra eigenvalue $\lambda_{0}=$ $-\sum_{\gamma=1}^{\Gamma} p^{\Gamma-\gamma} \lambda_{\gamma}$. For the translation-noninvariant Parisi matrix we generalize (3) in the way similar to the one used for block-hierarchical kinetic matrices (see [21]). Remind that for eigenvalues of kinetic matrix, one has

$$
\lambda_{\gamma, n}=-p^{\gamma} t_{\gamma}^{(n)}-\left(1-p^{-1}\right) \underbrace{\sum_{\gamma^{\prime}=\gamma+1}^{\Gamma} p^{\gamma^{\prime}} t_{\gamma^{\prime}}^{\left(n^{\prime}\right)}}_{\Sigma}
$$

i.e. the eigenvalue $\lambda_{\gamma, n}$ can be expressed via a linear combination ( $\Sigma$ in (44) of weighted matrix elements $t_{\gamma^{\prime}}^{\left(n^{\prime}\right)}$ coming from the vertices $\left(\gamma^{\prime}, n^{\prime}\right)$ along a unique path on the $p$-adic Cayley tree from the vertex $\left(\gamma^{\prime}=\gamma, n^{\prime}=n\right)$ towards the root vertex $\left(\gamma^{\prime}=\Gamma, n^{\prime}=1\right)$ - see 22] for more details. In the framework of same geometrical interpretation, the eigenvalue $\lambda_{\gamma, n}$ of the block-hierarchical matrix with $T_{i, i}=0$ (shown in Fig 1b) seems to be the linear combination of weighted matrix elements $t_{\gamma^{\prime}}^{\left(n^{\prime}\right)}$ along a path on the $p$-adic Cayley tree from the bottom level $\gamma^{\prime}=1$ to the vertex $\left(\gamma^{\prime}=\gamma, n^{\prime}=n\right)$. However this construction has an ambiguity since the corresponding path is not uniquely defined by the pair $(\gamma, n)$. So, rigorously speaking, the eigenvalues of particular realization of the random blockhierarchical adjacency matrix cannot be parameterized by the pairs $(\gamma, n)$, and we cannot write an exact expression for the eigenvalues in the form of expression (3). However for computation of spectral density, $\rho_{G}(\lambda)$, we use below $a$ posteriori self-averaging arguments, which make our consideration self-consistent. Moreover, the extensive numerical simulations confirm our analytic prediction of $\rho_{G}(\lambda)$ for $|\lambda| \gg 1$ in the interval $0<\nu<1$. Thus, formally extending (3) to the case of $\lambda_{\gamma, n}$ of block-hierarchical matrix $T$, we replace in (3) the first term by $t_{\gamma}^{(n)}$ and the sum - by $\sum_{\gamma^{\prime}=1}^{\gamma} p^{\gamma^{\prime}} t_{\gamma}^{\left(n^{\prime}\right)}$ where the summation runs now along the paths on the Cayley tree from the hierarchical level $\gamma^{\prime}=1$ to the vertex $(\gamma, n)$ located on the hierarchical level $\gamma^{\prime}=\gamma$ (compare to (44)). Supposing the distribution of the matrix elements $t_{\gamma}^{(n)}$ to be Gaussian,

$$
P\left(t_{\gamma}^{(n)}\right)=\frac{1}{\sqrt{\pi \sigma_{\gamma}^{2}}} \exp \left(-\frac{\left(t_{\gamma}^{(n)}\right)^{2}}{\sigma_{\gamma}^{2}}\right)
$$

and using for $\lambda_{\gamma, n}$ the guessed expression as a linear combination of matrix elements, we end up with the following 
equation for the spectral density, $\rho_{G}(\lambda)$ :

$$
\rho_{G}(\lambda)=p^{-\Gamma} \sum_{\gamma, n}\left\langle\delta\left(\lambda-\lambda_{\gamma, n}\right)\right\rangle_{P\left(t_{\gamma}^{(n)}\right)}=\frac{1}{\sqrt{\pi}} \sum_{\gamma=1}^{\Gamma} p^{-\gamma} \frac{1}{\sqrt{u_{\gamma}^{2}}} \exp \left(-\frac{\lambda^{2}}{u_{\gamma}^{2}}\right)
$$

where for $\sigma_{\gamma}=p^{-\nu \gamma}$ we have:

$$
u_{\gamma}^{2}=p^{2(\gamma-1)} \sigma_{\gamma}^{2}+\left(1-p^{-1}\right) \sum_{\gamma^{\prime}=1}^{\gamma-1} p^{2 \gamma^{\prime}} \sigma_{\gamma^{\prime}}^{2}=\frac{p-2}{p} p^{2(1-\nu) \gamma}+\frac{(p-1)^{2}}{p-p^{\nu}}\left(p^{2(1-\nu) \gamma}-1\right)
$$

For $p=2$ we can rewrite $\rho_{G}(\lambda)$ in (6) for $\Gamma \rightarrow \infty$ as follows

$$
\rho_{G}(\lambda) \simeq \frac{1}{\sqrt{\pi}} \sum_{\gamma=1}^{\infty} 2^{-(2-\nu) \gamma} \exp \left[-\lambda^{2} \frac{4-4^{\nu}}{2^{2(1-\nu) \gamma}-1}\right]
$$

Taking into account that

$$
\sum_{\gamma=1}^{\infty} p^{-c_{1} \gamma} e^{-t p^{-c_{2} \gamma}} \simeq t^{-c_{1} / c_{2}} \quad(t \gg 1)
$$

and substituting $c_{1}=2-\nu$ and $c_{2}=1-\nu$, we arrive at the following asymptotic form for the spectral density at $|\lambda| \gg 1:$

$$
\rho_{G}(\lambda) \simeq|\lambda|^{-\xi(\nu)} \quad(0<\nu<1)
$$

where

$$
\xi(\nu)=\frac{2-\nu}{1-\nu}
$$

The arguments supporting our derivation of the expression (77) for the spectral density $\rho_{G}(\lambda)$ are as follows. First of all, note that (6) - (7) become exact if we skip the dependence on $n$ in the matrix elements $t_{\gamma}^{(n)}$ and, hence, restore the translational invariance in the block-hierarchical matrix $T$. Secondly, we found in the extensive numeric simulations summarized in Fig 3 that indeed the conjectured behavior (10)-(11) actually holds for translation noninvariant Parisi matrices. In Fig 3 we have plotted the tails of the spectral density $\rho_{G}(\lambda)$ for Gaussian ensemble of block-hierarchical matrices for $N=256$. The solid and dot-dashed lines have the slopes $-\xi(\nu)$, where $\xi(\nu=0)=2$ (solid line) and $\xi(\nu=0.8)=6$ (dashed line). The scatter graphs from top to bottom correspond to $\nu=0.0,0.2,0.4,0.6,0.8,1.0$.

The fact that (10) gives right asymptotic behavior occurs apparently due to an effective self-averaging of the sum of matrix elements along each particular path on a Cayley tree for the distribution $\{\mathcal{S}\}=\left\{\sigma_{1}, \sigma_{2}, \ldots\right\}$. One sees that in the sum in (7) for $\sigma_{\gamma}=p^{-\nu \gamma}$ the lower limit of the summation can be shifted from $\gamma^{\prime}=1$ to $\gamma^{\prime} \rightarrow-\infty$. Asymptotically the result for $u_{\gamma}^{2}$ will remain unchanged if $0<\nu<1$. Such an extension of summation means that the computation of the spectral density (and, in particular, of $\lambda_{\gamma, n}$ ) involves the summation along the infinite paths running from $-\infty$ to the hierarchical level $\gamma$. We expect that for $\sigma_{\gamma}=p^{-\nu \gamma}(0<\nu<1)$ due to the convergence of the $\operatorname{sum} \sum_{\gamma^{\prime}=-\infty}^{\gamma} p^{2 \gamma^{\prime}} \sigma_{\gamma^{\prime}}^{2}$, the eigenvalue $\lambda_{\gamma, n}$ does not depend on each particular path on a Cayley tree and hence, the eigenvalue does not depend on the index $n$. Once this point of view is accepted, we return to an effective translation invariant block-hierarchical matrix for which (8) is exact.

One can see from (10)-(11) and Fig 3 that for $\nu \geq 1$ the power-law behavior of the spectral density $\rho_{G}(\lambda)$ terminates. This termination deserves special attention. Indeed, for $\nu \geq 1$ we cannot extend the lower limit of summation over $\gamma^{\prime}$ to $-\infty$ since the corresponding sum diverges. Hence, the contribution to the eigenvalues (and, therefore, to the spectral density) strongly depend on the particular configuration of the path. In this case the self-averaging, suggested in our computation, is invalid anymore and we cannot say anything about the behavior of the spectral density in the region $\nu \geq 1$. The opposite case $\nu<0$ deserves special attention because formally the sum (77) converges for any $\nu<0$ and our arguments about self-averaging seem to work. However the details of the analysis of this case is beyond the scope of the current work and will be discussed elsewhere.

Our approach to the construction of the networks with the scale-free behavior in the spectral density is not unique. As it is shown in the recent work 23], the procedure of dividing Gaussian matrices by a random variable, as well as the same procedure applied to random graphs, leads to the spectral density interpolating between the Erdös-Rényi and the scale-free models. 


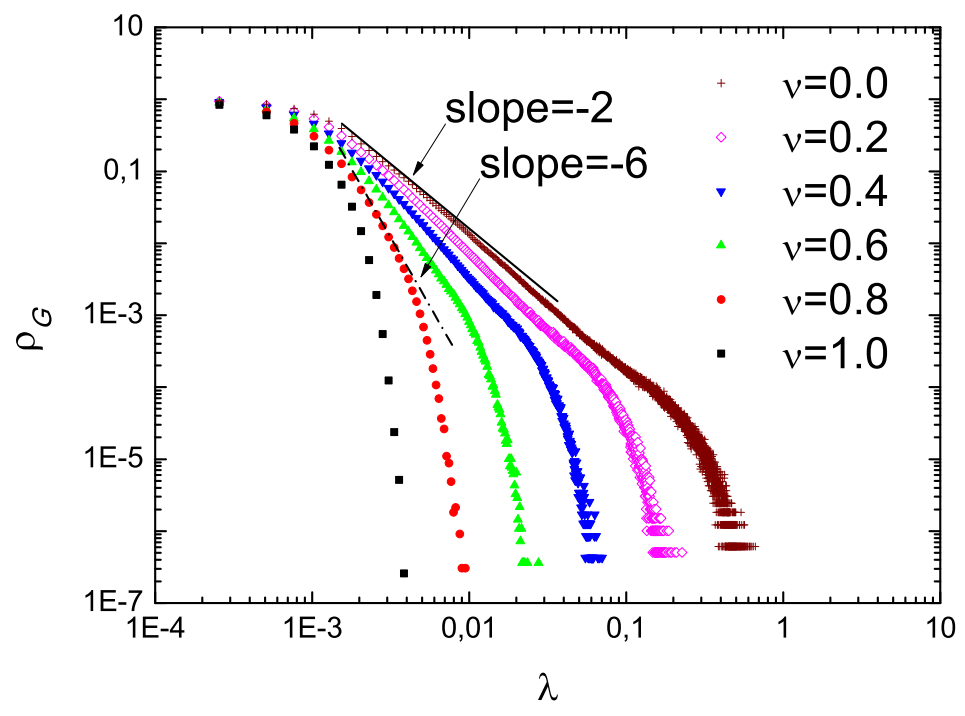

Figure 3: Spectral density for symmetric Gaussian translationary noninvariant block-hierarchical matrices. The solid and dot-dashed lines have the slopes $\xi(\nu=0)=2$ and $\xi(\nu=0.8)=6$; scatter graphs: $\nu=0(+), \nu=0.2(\diamond), \nu=0.4(\boldsymbol{\nabla}), \nu=0.6$ $(\boldsymbol{\Delta}), \nu=0.8(\bullet), \nu=1.0(\mathbf{\square})$.

\section{HIERARCHICAL "GROWING" OF POLY-SCALED NETWORKS}

One can roughly distinguish two methods of construction the scale-free networks. The first method has been developed mainly for illustrative purposes and deals with the hierarchical construction 24] of deterministic scale-free graphs with predetermined fractal properties such as, say, vertex degree distribution. Since in this case the obtained graphs are deterministic, it is senseless to talk about any statistics of their spectra. The second method deals with the variants of iterative "preferential attachment" construction [25], where new nodes are added to a vertex of the network with the probability depending on already existing vertex degree (the number of one-step connections to other vertices). Almost all known statistical characteristics of scale-free networks, including the spectral density of adjacency matrix, are obtained for the networks constructed using this method. Typically, the spectral density of the ensemble of scale-free networks designed by the preferential attachment method has a triangle-like shape in the "bulk" part with power-law tails.

Note that the construction of scale-free networks by preferential attachment method is based on locally nonuniform incremental growth with unlimited evolutionary memory. In contrast to this, we propose below another physically motivated approach to the construction of the random networks with power-law spectral density by a "parallel" (i.e. non-stepwise) and uniform procedure.

The peculiarity of our construction consists in the following. We build clusters of edges with hierarchically organized probabilities, while the typical procedure consists in hierarchical grouping of vertices. Our procedure does not impose any additional metric structure on the graph and leaves the graph (network) purely topological. To the contrary, grouping of vertices imposes a metric structure on the graph (network) since such a grouping operates usually with the notion of "close" (or "distant") vertices.

To shed light on spectral properties of random hierarchical graphs we exploit the link between the Random Matrix Theory (RMT) and the Graph Theory (GT). It is known that the spectral density $\rho(\lambda)=$ $\frac{1}{N} \sum_{i=1}^{N}\left\langle\delta\left(\lambda-\lambda_{i}\right)\right\rangle_{\left\{q_{1}, q_{2}, \ldots, q_{\Gamma}\right\}}$ of the ensemble of adjacency matrices is directly related to the topological structure of the corresponding network since the value

$$
M_{k}=\frac{1}{N} \int \lambda^{k} \rho(\lambda) d \lambda=\frac{1}{N} \sum_{i=1}^{N} \lambda_{i}^{k}
$$

defines (up to the factor $N$ ) the average number of $k$-step loops in the network (see, for example, [18]).

In particular, in [26] it has been shown that in the thermodynamic limit the spectral properties of random ErdösRényi graphs [27] coincide with the spectral properties of random real symmetric matrices. This result is one of 
the benchmarks in our consideration. The elements of adjacency matrix, $A_{i, j}$, of Erdös-Rényi graph are Bernoullidistributed random variables: $A_{i, j}=1$ or 0 with probabilities $q$ and $1-q$ correspondingly. For the ensemble of ER-random graphs the density, $\rho_{A}(\lambda)$, of eigenvalues of adjacency matrices $A$ can be analytically computed in the thermodynamic limit $N \rightarrow \infty$ and has for some $q$ a celebrated Wigner-Dyson semicircle law known for ensembles of Gaussian matrices [28]. Namely, the following statement is proved [26]. Let $B$ be a real symmetric $N \times N$ matrix with independently distributed entries $B_{i, j}$ from, say, Gaussian distribution $P\left(B_{i, j}\right)$ with $\left\langle B_{i, j}\right\rangle=0$ and $\left\langle B_{i, j}\right\rangle=\sigma^{2}$. Then the spectral density, $\rho_{B}(\lambda)$, of the ensemble of matrices $B$ converges in the limit $N \rightarrow \infty$ to the semicircle distribution

$$
\rho_{B}(\lambda)= \begin{cases}\frac{1}{2 \pi \sigma^{2}} \sqrt{4 N \sigma^{2}-\lambda^{2}} & \text { if }|\lambda|<\sqrt{4 N \sigma^{2}} \\ 0 & \text { if }|\lambda|>\sqrt{4 N \sigma^{2}}\end{cases}
$$

If $\sigma^{2}=q(1-q)$, then $\rho_{A}(\lambda)=\rho_{B}(\lambda)$ for $N \rightarrow \infty$, i.e. the spectral densities of ensembles of random ER-graphs and of Gaussian symmetric matrices coincide in the thermodynamic limit. Nevertheless, such a coincidence of spectral densities for random graphs and random Gaussian matrices should not be understood in a literal sense: some spectral properties of random ER-graphs and random matrices are different [18, 29]. For example, since for the adjacency matrix $A$ of random ER-graphs one has $\left\langle A_{i, j}\right\rangle=q$, then the corresponding largest eigenvalue, $\lambda_{1}$, grows linearly with the system size, $N$, i.e. $\lambda_{1}=N q$, meaning that the semicircular distribution for random graphs is valid only for the matrix $A-\langle A\rangle$. Also, the tails of spectral distributions near the spectrum edges are different for random graphs and random matrices. Nevertheless, as a first approximation, the random Gaussian matrices could serve as a very natural benchmark for the corresponding statistical analysis. The comparison of spectral properties of hierarchical and random Erdös-Rényi graphs is demonstrated in Fig,4. The Fig 4 a shows the semi-log plot of the spectral densities for: (i) random hierarchical graphs with LCP adjacency matrices for $N=256$ and $\mu=0.2$ (solid line), and (ii) random

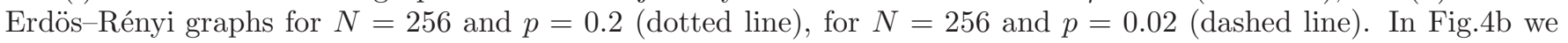
have redrawn the central part of Fig $4 \mathrm{a}$ in the linear scale.

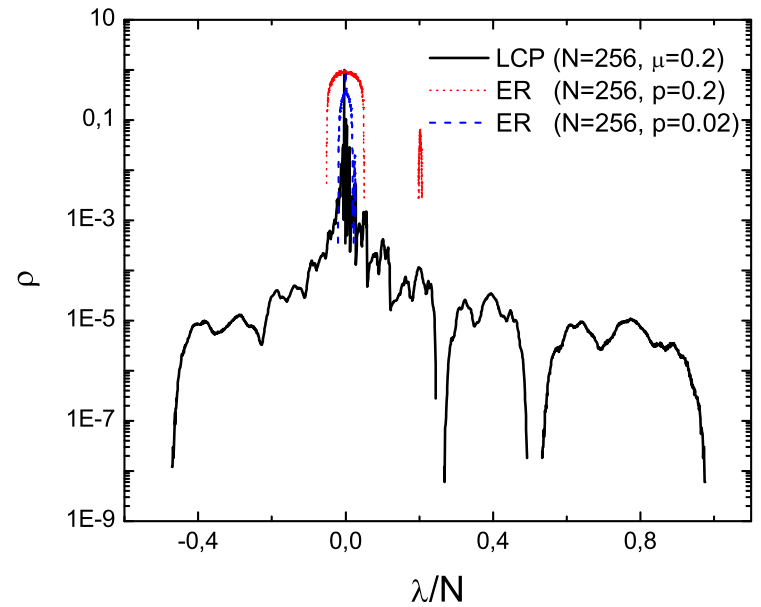

(a)

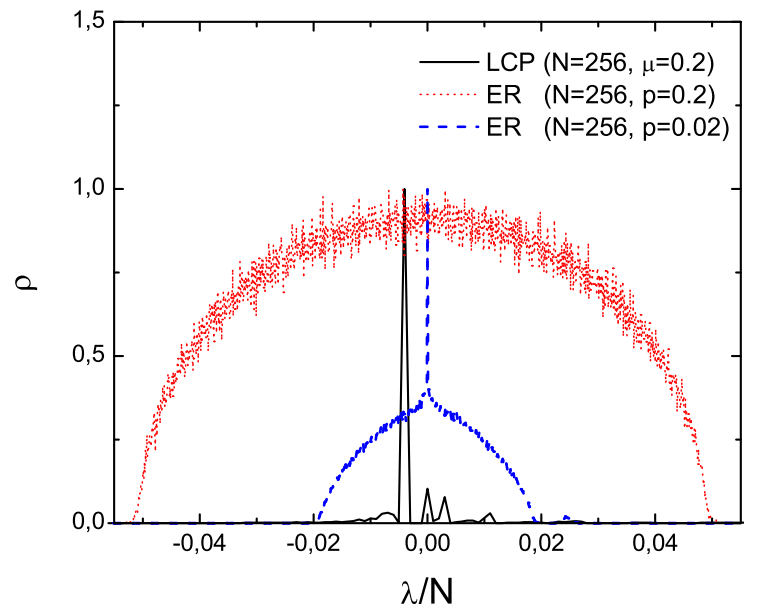

(b)

Figure 4: Comparison of spectral densities of random block-hierarchical matrix and Erdös-Rényi (ER) random graphs: (a) in semi-logarithmic coordinates in the region $-0.5<\lambda / N<0.5$; and (b) in linear coordinates in the region $-0.05<\lambda / N<0.05$.

The numerical results for the probability distributions $P\left(M_{k}\right)(k=2,3)$ of the number of $k$-step loops in random hierarchical graphs for $N=256$ and $\mu=0.2$ are compared in Fig 5 with the distributions of loops on Erdös-Rényi random graphs for $N=256$ and $p=0.2$. Recall that $P\left(M_{2}\right)$ defines the probability to have in the finite graph the average connection degree equal to $M_{2}$.

One sees that the distribution functions $P\left(M_{2}\right)$ and $P\left(M_{3}\right)$ for our hierarchical random graphs are much broader than the corresponding distributions for Erdös-Rényi graphs with the same number of vertices. Hence, the topological structure of random hierarchical graphs is much more "flexible" than that of random ER-graphs. This is consistent with the found behavior for the spectral density: the distribution function $\rho(\lambda)$ has "heavy" tails and decays much slower than the that for random ER-graphs. According to the behavior of the distribution functions $P\left(M_{2}\right)$ and $P\left(M_{3}\right)$ it is naturally to call our random block-hierarchical graph the "poly-scaled". 

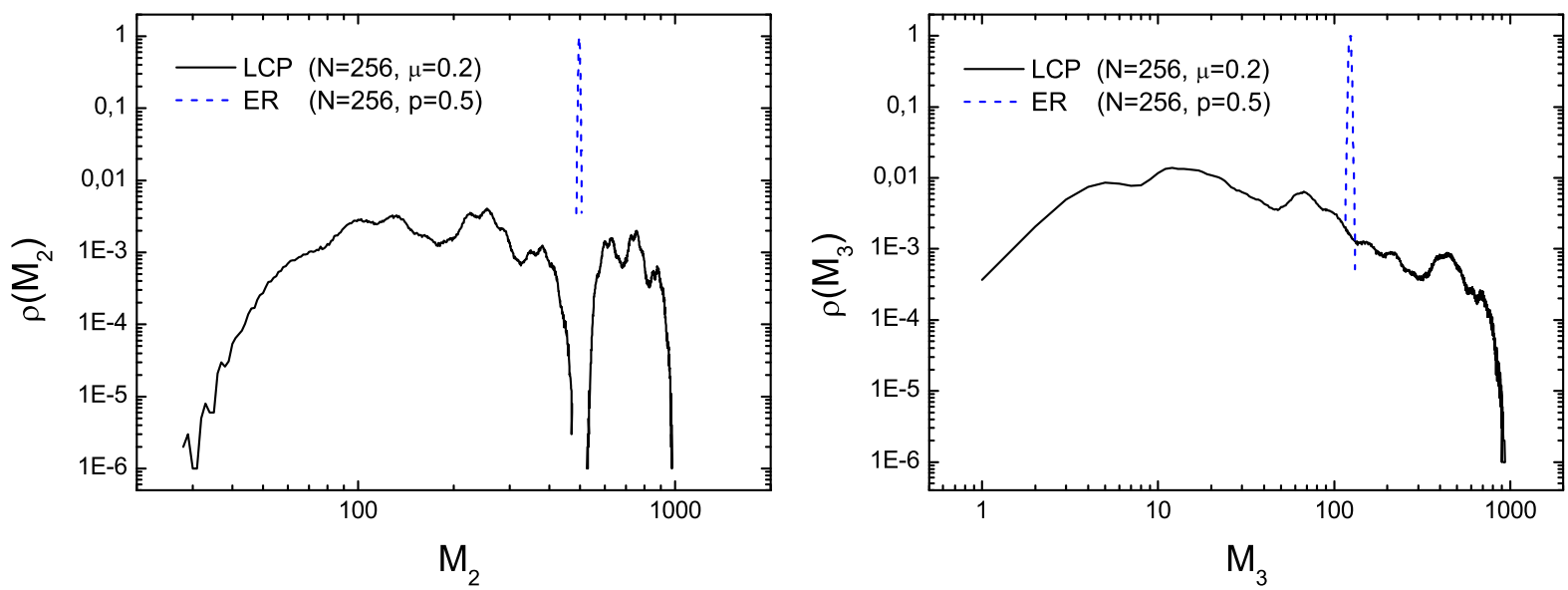

Figure 5: Distribution of the number of 2- and 3-step loops in random hierarchical and Erdös-Rényi graphs.

The method of generating functions allows us to compute easily the vertex degree distribution in the ensemble of random block-hierarchical graphs directly by their adjacency matrices. To do that let us consider any (for example, the first) row in the adjacency matrix $T$ (see Fig 1 ). The total number of links of the first graph vertex to other vertices is defined by the number of matrix elements, $t_{\gamma}^{(1)}(\gamma=1, \ldots, \Gamma)$, having in the first row nonzero value (i.e. taking the value "1"). Thus, the distribution of the number of connections (i.e. vertex degree distribution), $\mathcal{P}(m)$, is the probability of the fact that the sum of matrix elements in the first row is exactly equal to $m$ under the condition that the matrix elements are grouped in the hierarchical blocks and have the binomial distributions $\left\{q_{1}, q_{2}, \ldots, q_{\Gamma}\right\}$ as it is defined in (1). Finally we arrive at the following expression for the degree distribution $\mathcal{P}(m)$ (in the sake of simplicity we have denoted $\left.q_{\gamma}\left(t_{\gamma}^{(1)}\right) \equiv q_{\gamma}\left(t_{\gamma}\right)\right)$ :

$$
\mathcal{P}(m)=\sum_{\left\{t_{1} \ldots t_{\Gamma}\right\}}\left[\prod_{\gamma=1}^{\Gamma} q_{\gamma}\left(t_{\gamma}\right)\right] \Delta\left(\sum_{\gamma=0}^{\Gamma} p^{\gamma} t_{\gamma+1}-m\right)
$$

where the binomial distributions $q_{\gamma}\left(t_{\gamma}\right)$ have the form

$$
q_{\gamma}\left(t_{\gamma}\right)=p^{-\mu \gamma} \delta_{q_{\gamma}\left(t_{\gamma}\right), 1}+\left(1-p^{-\mu \gamma}\right) \delta_{q_{\gamma}\left(t_{\gamma}\right), 0}
$$

and $\Delta(\ldots)$ is the Kronecker symbol:

$$
\Delta(x)=\frac{1}{2 \pi i} \oint d z z^{x-1}= \begin{cases}1 & \text { if } x=0 \\ 0 & \text { if } x \neq 0\end{cases}
$$

Substituting (15) and (16) in (14) after elementary transformations we get

$$
\mathcal{P}(m)=\frac{1}{2 \pi i} \oint d z z^{-(m+1)} \prod_{\gamma=1}^{\Gamma} W(z, \gamma)
$$

where

$$
W(z, \gamma)=p^{-\mu \gamma} z^{p^{\gamma}}+1-p^{-\mu \gamma}
$$

For not too large values of $\Gamma$ the distribution $\mathcal{P}(m)$ can be analyzed numerically. Using the fact that the function $W(z, \gamma)$ is a polynomial of $z$, let us represent $\mathcal{P}(m)$ as:

$$
\mathcal{P}(m)=\left.\frac{1}{(m+1) !} \frac{d^{m+1}\left[\prod_{\gamma=1}^{\Gamma} W(z, \gamma)\right]}{d z^{m+1}}\right|_{z=0}
$$

In Fig $[6$ a we have depicted the family of curves $\mathcal{P}(m)$ for $\Gamma=16$ and $\mu=0.1 ; 1.0$. For comparison, in Fig 6 . we have plotted the distribution $\mathcal{P}(m)$ for $\mu=1.0, \Gamma=16$ as well as the binomial distribution $\mathcal{P}_{\mathrm{ER}}(m)=C_{N}^{m} q^{m}(1-q)^{N-m}$ 


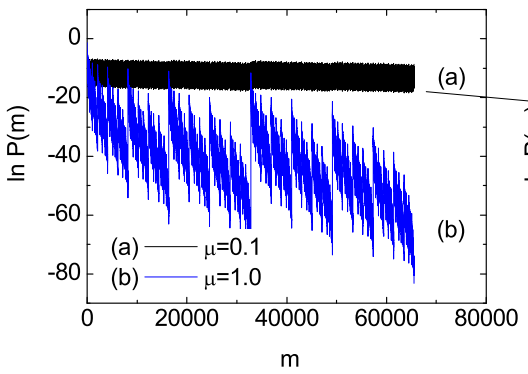

(a)

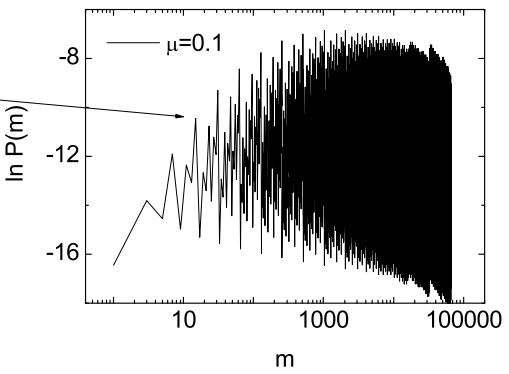

(b)

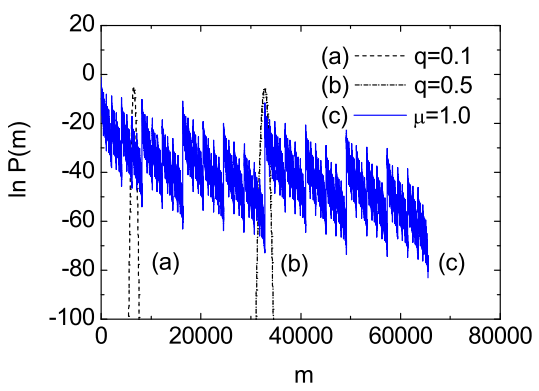

(c)

Figure 6: a) The family of distributions $\mathcal{P}(m)$ for $\Gamma=16$ at $\mu=0.1 ; 1.0 ;$ b) Distribution $\mathcal{P}(m)$ in the double-logarithmic scale for $\mu=0.1$ and $\Gamma=16$; c) Comparison of $\mathcal{P}(m)$ for the random block-hierarchical graph $(\mu=1.0, \Gamma=16)$ and $\mathcal{P}_{\mathrm{ER}}(m)$ for the Erdös-Rényi graph $\left(q=0.1 ; 0.5, N=2^{16}\right)$.

for the standard Erdös-Rényi graph with the number of vertices $N=2^{\Gamma}=2^{16}$ and for two values $q=0.1 ; 0.5$. One can see on Fig 6. how wider is the distribution $\mathcal{P}(m)$ with respect of the corresponding distribution $\mathcal{P}_{\mathrm{ER}}(m)$ for ER-graphs.

The fractal structure of the distribution $\mathcal{P}(m)$ for random hierarchical networks demonstrated in Fig 6 a,b, apparently is deeply linked to the invariant multifractal measures appearing in chaotic Hamiltonian systems in connection with the problems of "number-theoretical chaos" - see, for example, [30]. Actually, the condition $\Delta\left(\sum_{\gamma=1}^{\Gamma} p^{\gamma} t_{\gamma}-m\right)$ in equation (14) for $\Gamma \rightarrow \infty$ is nothing else as the binary expansion of the number $m$ : $m=t_{1} 2^{0}+t_{2} 2^{1}+t_{3} 2^{2}+\ldots+t_{\gamma+1} 2^{\gamma}+\ldots$, where the coefficients $t_{\gamma}$ take values 1 or 0 with corresponding probabilities $q_{\gamma}\left(t_{\gamma}\right)$ defined in (15). Let us note that the similar expansion of the form $\sum_{k=1}^{\infty} \varepsilon_{k} u^{-k}$, where $u>1$ and $\varepsilon_{k}= \pm 1$ (with equal probabilities $\frac{1}{2}$ independent of $k$ ) is known in the literature as the "singular Erdös measure" [31, 32, 33]. The observed fractal structure of the distribution function $\mathcal{P}(m)$ appears due to the effects of the incommensurability of number-theoretic origin: some binary expansions (of the number $m$ ) with random coefficients have relatively high probabilities to appear in the adjacency matrix, while other binary expansions have much less possibilities.

\section{CONCLUSION}

First of all, let us note that our consideration of spectral properties of random hierarchical graphs is far from being complete and many other properties are of interest (for example, eigenvectors, inverse participation ratio, etc.- see, for instance [19]). However even in this preliminary investigation we would like to emphasize the crucial difference between random hierarchical and random Erdös-Rényi graphs.

Roughly speaking, there are two generic ways of the scale-free network construction. The similarities and differences of these two approaches we would like to emphasize below again.

- The first way, widely discussed in the literature, deals basically with the "preferential attachment" procedure, where the network is raised by the essentially non-Markovian (in the increments) evolution process with unlimited memory. The evolutionary process of such kind can be viewed, to some extent, as a "generalized Brownian motion". The realization of such a procedure demands the monitoring of the whole network structure on each step, because the appearance of new links depends on the current degree of graph vertices. From this point of view the sequential construction of the corresponding network can be tentatively denoted as a "nonlinear evolution".

- The second way, discussed in the present work, exploits essentially different mechanism of the scale-free network formation. The hierarchical organization of probabilities of links in the topological network constitutes the basic idea of our construction. Specifically, we construct the networks with the scale-free eigenvalues distribution of the adjacency matrices, where the last originates from an appropriate randomization of the standard Parisi matrix - one of the key objects in the theory of spin glasses. Our method allows one to build a hierarchical network in a memoryless locally uniform way. The application of the elements of the $p$-adic analysis 34 permit us to analyze the basic spectral properties of ensembles of randomized Parisi-type adjacency matrices. 
To end up, let us emphasize that there are two important features of hierarchical networks constructed in our paper. First of all, any sub-graph belonging to particular hierarchy level, $\gamma$, is just a random Erdös-Rényi graph because the formation of clusters of bonds on each hierarchy level is entirely uncorrelated. Secondly, the random sub-graphs, associated with different hierarchy levels of the network, can be different, so the network in a whole can be essentially nonuniform. Nevertheless as we have seen, the "mipmapping" construction with different sets of parameters (i.e. the hierarchical embeddings of sub-graphs corresponding to the different hierarchical levels) leads under some conditions to the scale-free behavior in the spectral density of adjacency matrix and to the poly-scale (and even to the fractal) behavior in various topological characteristics of the graph. This observation is rather unexpected since in our case the scale-free behavior is reached by essentially Markovian and memoryless procedure.

In physics the random graphs of such a hierarchical genesis can be encountered among the scale-free networks whose natural origin are associated with low-correlated random events carried out under short evolutionary memory. In particular, the networks of hierarchical genesis may by interesting for the prebiology or the earliest biology.

\section{Acknowledgments}

We are grateful to K. Bashevoy and A.Bikulov for illuminating numerical simulations and to O. Bohigas and Y. Fyodorov for helpful discussions. S.K.N. is indebted to Z. Toroczkai for stimulating discussion of the topic on initial stages of the work. This work is partially supported by the RFBR grant No. 07-02-00612a.

[1] P. Erdös, A. Rényi, Publ. Math. Inst. Hung. Acad. Sci. Ser. A 517 (1960)

[2] G.J. Rodgers, K.Austin, B.Kahng, D.Kim, J. Phys. A: Math. Gen. 389431 (2005)

[3] E. Ben-Naim, P.L. Krapivsky, Phys. Rev. E 71026129 (2005)

[4] R. Albert and A.-L. Barabási, Rev. Mod. Phys., 7447 (2002)

[5] M. Mezard, G. Parisi, M. Virasoro, Spin glass theory and beyond (World Scientific: Singapore, 1987)

[6] B.V. Chirikov, D.L. Shepelyansky, Physica D 13395 (1984)

[7] J.D. Meiss, E. Ott, Physica D 20387 (1986)

[8] A.V. Dobrynin, M. Rubinstein, S.P. Obukhov, Macromolecules 292974 (1996)

[9] P.W. Fenimore, H. Frauenfelder, B.H. McMahon, R.D. Young, Physica A 3511 (2005)

[10] I. M. Lifshitz, J. Eur. Theor. Phys. 552408 (1968)

[11] I. M. Lifshits, A. Y. Grosberg and A. R. Khokhlov, Rev. Mod. Phys. 50683 (1978)

[12] A. Y. Grosberg and A. R. Khokhlov, Statistical Physics of Macromolecules (AIP Press: New York, 1994)

[13] J. des Cloizeaux and G. Jannink, Polymers in Solution: Their Modelling and Structure (Oxford University Press: New York, 1990)

[14] A. Y. Grosberg, S. K. Nechaev and E. I. Shakhnovich, J. Phys. (Paris) 492095 (1988)

[15] S. Nechaev, O. Vasilyev, J. Knot Theory Ramific. 14, 243 (2005); S. Nechaev, O. Vasilyev, in Physical and Numerical Models in Knot Theory, Ch. 22, p. 421, Series on Knots and Everything, (WSPC: Singapore, 2005)

[16] A. Grosberg, Y. Rabin, S. Havlin, A. Neer, Europhys. Lett. 23373 (1993)

[17] A.J. Einstein, H.-S. Wu, J. Gil, Phys. Rev. Lett., 80397 (1998)

[18] I.J. Farkas, I. Derényi, A.-L. Barabási, T. Vicsek, Phys. Rev. E 64026704 (2001)

[19] K.-I. Goh, B. Kahng, D. Kim, Phys. Rev. E 64051903 (2001)

[20] A. Ogielsky, D. Stein, Phys. Rev. Lett. 551634 (1985); C.P. Bachas, B. A. Huberman, Phys. Rev. Lett. 57 1965 (1986)

[21] V. A. Avetisov, A. Kh. Bikulov, S. V. Kozyrev, V. A. Osipov J. Phys. A: Math. Gen. 35177 (2002)

[22] V.A. Avetisov, A.Kh. Bikulov, S.K. Nechaev, J. Phys. A: Math. Theor. 42075001 (2009)

[23] O. Bohigas, J.X. de Carvalho, M.P. Pato, Phys. Rev. E 77011122 (2008)

[24] A.-L. Barabási, E. Ravasz, and T. Vicsek, Physica A 299559 (2001).

[25] A.-L. Barabási and R. Albert, Science 286509 (1999); A.-L. Barabási R. Albert, H. Jeong, Physica A 272173 (1999); A.-L. Barabási, R. Albert, H. Jeong, Physica A 28169 (2000)

[26] F. Juhász, in Algebraic methods in graph theory, Coll. Math. Soc. J. Bolyai 25, 313 (North-Holland, 1981); Z. Füredi, J. Komlós, Combinatorica 1233 (1981)

[27] P. Erdös, A. Rényi, Publ. Math. Inst. Hung. Acad. Sci. 517 (1960)

[28] F.J. Dyson, J. Math. Phys. 3, 140 (1962); E.P. Wigner, SIAM Rev. 91 (1967)

[29] I. Farkas, I. Derényi, H. Jeong, Z. Néda, Z.N. Oltvai, E. Ravasz, A. Schubert, A.-L. Barabási, T. Vicsek, Physica A 314 $25(2002)$

[30] M.C. Gutzwiller, B.D. Mandelbrot, Phys. Rev. Lett. 60673 (1988)

[31] P. Erdös, Amer. J. Math. 61974 (1939)

[32] B. Solomyak, Annals of Mathematics, 142611 (1995) 
[33] N. Sidorov, A. Vershik, Monatshefte fü̈ Mathematik, 126215 (1998)

[34] V. A. Avetisov, A. Kh. Bikulov, S. V. Kozyrev, J. Phys. A: Math. Gen. 328785 (1999); G. Parisi, N. Sourlas, Eur. J. Phys. B 14535 (2000) 